\title{
On the computation of stable sets and strictly perfect equilibria
}

Citation for published version (APA):

Vermeulen, A. J., \& Jansen, M. J. M. (2001). On the computation of stable sets and strictly perfect equilibria. Economic Theory, 17, 325-344. https://doi.org/10.1007/PL00004106

Document status and date:

Published: 01/01/2001

DOI:

10.1007/PL00004106

Document Version:

Publisher's PDF, also known as Version of record

\section{Please check the document version of this publication:}

- A submitted manuscript is the version of the article upon submission and before peer-review. There can be important differences between the submitted version and the official published version of record.

People interested in the research are advised to contact the author for the final version of the publication, or visit the DOI to the publisher's website.

- The final author version and the galley proof are versions of the publication after peer review.

- The final published version features the final layout of the paper including the volume, issue and page numbers.

Link to publication

\footnotetext{
General rights rights.

- You may freely distribute the URL identifying the publication in the public portal. please follow below link for the End User Agreement:

www.umlib.nl/taverne-license

Take down policy

If you believe that this document breaches copyright please contact us at:

repository@maastrichtuniversity.nl

providing details and we will investigate your claim.
}

Copyright and moral rights for the publications made accessible in the public portal are retained by the authors and/or other copyright owners and it is a condition of accessing publications that users recognise and abide by the legal requirements associated with these

- Users may download and print one copy of any publication from the public portal for the purpose of private study or research.

- You may not further distribute the material or use it for any profit-making activity or commercial gain

If the publication is distributed under the terms of Article $25 \mathrm{fa}$ of the Dutch Copyright Act, indicated by the "Taverne" license above, 


\title{
On the computation of stable sets and strictly perfect equilibria
}

\author{
Mathijs Jansen and Dries Vermeulen \\ Department of Quantitative Economics, Maastricht University, P.O. Box 616, \\ 6200 Maastricht, THE NETHERLANDS (e-mail: m.jansen@ke.unimaas.nl)
}

Recieved: December 29, 1999; revised version: February 17, 2000

Summary. In this paper a procedure is described that computes for a given bimatrix game all stable sets in the sense of Kohlberg and Mertens (1986). Further the procedure is refined to find the strictly perfect equilibria (if any) of such a game.

Keywords and Phrases: Bimatrix game, Computation, Stable equilibrium, Strictly perfect equilibrium.

JEL Classification Numbers: C72, C73.

\section{Introduction}

History. An $n$-person (noncooperative) game is a game in which each player has to choose one of his (finite number of) pure strategies without any prior knowledge of what the other players are going to do. Given the choices of all players each player receives a payoff. The players are also allowed to mix over pure strategies, i.e. to use probability distributions over pure strategies to determine their respective choices. Payoffs are then determined as expected payoffs with respect to the mixtures employed by the players. Nash (1950) showed that such games always have at least one equilibrium.

Since then it has become clear that this notion of Nash equilibrium is not the final answer to the problem of solving games. It is for instance well known that a Nash equilibrium may use weakly dominated strategies (a strategy is called weakly dominated if there is another strategy that does at least as good as this strategy against any combination of strategies of the opponents and strictly better 
against at least one). This observation, along with some other points of critique ${ }^{1}$, triggered a whole series of papers presenting different types of selection criteria for Nash equilibria. Generally speaking the idea was to find a solution concept ${ }^{2}$ that weeded out the "bad" Nash equilibria.

(Although there is also a line of literature that tries to weaken the equilibrium conditions these notions usually address the coordination problem involved in equilibrium selection. The Nash conditions themselves are hardly ever questioned.)

Basically we can distinguish two lines of research within equilibrium selection theory. The first form of selection is known as refinement theory. Examples are perfect equilibrium by Selten (1975), proper equilibrium by Myerson (1978) and strictly perfect equilibrium by Okada (1981). These refinements were all designed in order to mend a newly discovered flaw of either the original notion of Nash equilibrium or of one of its previously defined refinements. The second stream of literature is commonly known as the theory of stable sets. Its most renowned exponents are defined in Kohlberg and Mertens (1986), Mertens $(1989,1991)$ and Hillas (1990). This line of research started with the seminal paper of Kohlberg and Mertens (1986). They argued that the theory of refinements lacked a fundamental basis. As we already said, most refinements were meant as patching-up jobs for known solution concepts. Kohlberg and Mertens took a different approach. They started with a list of desiderata that were in their view essential for any decent solution concept. Only then they started to search for solution concepts that actually satisfied the desiderata. This quest more or less ended with the papers of Mertens $(1989,1991)$ in which he presented a definition of stable sets that he argued to be essentially the right concept.

Computation. For several reasons it is desirable to have an algorithm that computes a given notion of equilibrium selection. First of all an algorithm shows that the notion is more than a theoretical tool. An algorithm unambiguously shows that it is always possible (at least within the domain considered) to actually compute the equilibrium for the given game. Besides that an algorithm can be implemented on a computer, which gives us the opportunity to analyze fairly large and complicated examples.

There is extensive literature on the computation of Nash equilibrium and its selections. Algorithms for the computation of Nash equilibria of bimatrix games can for example be found in Lemke and Howson (1964), Winkels (1979) and Krohn et al. (1991). These algorithms are implementable and exact ${ }^{3}$. A general $n$-person version of the Lemke-Howson approach is described in Rosenmüller (1971). This procedure is not directly implementable though. Implementable algorithms (based on path-following algorithms for roots of homotopies) for the

\footnotetext{
${ }^{1}$ For an elaborate discussion of the issues involved here we refer to Kohlberg and Mertens (1986).

2 A solution concept is a rule that assigns to each game a collection of (sets of) strategy combinations of that game.

${ }^{3}$ An algorithm is called exact in this case if, given the data of the game, it takes a finite number of operations to exactly produce a strategy for each player that together define a (selection of) Nash equilibrium.
} 
computation of a Nash equilibrium for $n$-person games are developed in van den Elzen and Herings (1999) and Herings and Peters (1999).

Concerning the computation of equilibrium selection, Yamamoto (1993) describes a path-following procedure for the computation of a proper equilibrium. This procedure is not fit for implementation though. Talman and Yang (1994) describe an algorithm for the computation of a proper equilibrium that is implementable. Van den Elzen and Talman (1991) provide an implementable algorithm for computation of a perfect equilibrium. Wilson (1992) devised an algorithm for computation of what he calls a simply stable set. Finally Mertens (1989) provides an idea of how to construct an algorithm for computation of his type of stable sets.

Why bimatrices. Many solution concepts for $n$-person games can be defined as the solution set of a number of polynomial (in)equalities (see for instance Blume and Zame, 1994). One way to exploit this fact is explained by Mertens (1989). He describes an algorithm that, given an $n$-person game, defines a finite number of systems of polynomial inequalities whose solution sets (i.e. the union over all systems of the solution sets of those systems) is a stable set in the sense of the definition given in Mertens (1989). Such an approach can also be devised for the computation of, for example, Nash equilibria, i.e. it is possible to find in finite time a (finite) set of polynomial inequalities whose solutions are Nash equilibria.

However, in this paper we will show that it is possible to find a description of the solution concepts in question in terms of the combinatorial structure underlying these solutions. In fact we will exploit the construction used by Jansen et al. (1994) in their proof of the finiteness of KM-stable sets. The example in Hillas et al. (1997) clearly shows that this construction does not extend to games with more than two players. Even though it is possible to construct a finite algorithm in the above sense to compute stable sets, only for bimatrix games is it possible to actually solve these inequalities.

Aim of the paper. In this paper we will focus on strictly perfect equilibria, defined by Okada (1981) and what we will call KM-stable sets, defined by Kohlberg and Mertens (1986). Kohlberg and Mertens (1986) considered the type of perturbations introduced by Selten (1975) where every player has to play each of his pure strategies with at least a specific minimal weight. Such a perturbation of the game can itself also be viewed as a game in which the players have less options than in the original game (in fact the perturbation can be interpreted as a restriction on the strategy space). Moreover, it can be shown that these perturbed games also have equilibria. Now Kohlberg and Mertens define a KM-set of the game as a closed set of Nash equilibria for which each sufficiently small perturbation of the game has an equilibrium close to the set itself. A KM-set is called KM-stable if it does not contain another KM-set (i.e., it is minimal w.r.t. the stability condition). Okada's notion of strictly perfect equilibrium now coincides with one-point KM-sets (and hence KM-stable sets. KM-sets that consist of one point are automatically stable). 
It is our aim to describe a procedure that computes a KM-stable set for a given bimatrix game. Further, we will explain how the central algorithm can also be used to compute all KM-stable sets of that game. Because of the relation between the notion of strictly perfect equilibrium and KM-stable set, this enables us to compute a strictly perfect equilibrium (or all of them).

The algorithm. Presenting our exact algorithm we basically follow the paper of Jansen et. al (1994). They showed that with each strategy pair $(p, q)$ we can associate a quadruple of subsets of the collections of pure strategies, namely the carriers of $p$ and $q$ and the sets of pure best replies against $p$ and $q$, respectively. Given this quadruple it is possible to calculate precisely against which perturbations of the game the strategy pair $(p, q)$ offers protection ${ }^{4}$. In fact, any other strategy pair that yields the same quadruple will offer the same protection.

This observation lies in the heart of the algorithm. Given a finite set of such quadruples we can calculate the associated sets of perturbations against which (any set of strategy pairs generating) these quadruples ${ }^{5}$ offers protection. This paper shows that one can check in finite time whether or not this collection of associated sets of perturbations covers the whole perturbation space.

We exploit this result in several ways. First of all, it enables us to calculate precisely which collections of quadruples generate a minimal cover of the perturbation space (minimal meaning that no proper subcollection of quadruples generates a cover of the perturbation space). Further, given a set of which we already know that it is a KM-set (such as the collection of all extreme Nash equilibria by Jansen et al. (1994)) the above construction provides a method to select a KM-stable set within the KM-set given. Finally, since a strictly perfect equilibrium is simply a one-point KM-stable set, the computation of all KMstable sets also provides a way to calculate all strictly perfect equilibria. There is also a procedure to compute just one strictly perfect equilibrium (if any) that operates a bit more subtle. Since any bimatrix game that has a strictly perfect equilibrium also has an extreme Nash equilibrium that is strictly perfect, it is possible to check in finite time whether there is such an equilibrium as soon as we can find all extreme Nash equilibria. This however has already been done in e.g. Winkels (1979).

Notation. If $M$ is a finite set, $|M|$ denotes the number of elements of $M$ and $\Delta_{M}:=\left\{p \in \mathbb{R}^{M} \mid p_{i} \geq 0\right.$ for all $i \in M$ and $\left.\sum_{i \in M} p_{i}=1\right\}$. For an $i \in M$, $e_{i}$ is the $i$-th unit vector in $\mathbb{R}^{M}$. For a set $S$ in $\mathbb{R}^{n}$, $\operatorname{ext}(S)$ denotes the set of extreme points of $S$. For a vector $x$ in $\mathbb{R}^{n}$ and $\zeta>0,\|x\|$ denotes the maximum norm and $B_{\zeta}(x):=\left\{y \in \mathbb{R}^{n} \mid\|x-y\|<\zeta\right\}$. For a set $S$ in $\mathbb{R}^{n}$ and $\zeta>0$, $B_{\zeta}(S):=\bigcup_{x \in S} B_{\zeta}(x)$. The Hausdorff distance $d_{H}(S, T)$ between two sets $S$ and $T$ in $\mathbb{R}^{n}$ is the infimum over all $\zeta>0$ such that $S \subset B_{\zeta}(T)$ and $T \subset B_{\zeta}(S)$.

\footnotetext{
${ }^{4}$ This is very typical for bimatrix games. It is possible to associate with the given strategy pair a maximal set of perturbations such that any sufficiently small perturbation within this set has an equilibrium close to $(p, q)$. In fact this is the second reason to restrict ourselves to bimatrix games.

${ }^{5}$ Of course the quadruples themselves do not offer protection against perturbations. However, since strategy pairs that generate the same quadruples offer protection against the same perturbations, it is possible, even convenient, to think in these terms.
} 
A collection $\mathscr{C}$ of sets is said to cover a set $D$ if $D$ is a subset of the union $\cup_{C \in \mathscr{C}} C$ over all sets $C$ in $\mathscr{C}$.

\section{Preliminaries}

In this paper we only consider bimatrix games. So we assume that there are two players, player 1 and player 2. Player 1 has a finite set $M$ and player 2 has a finite set $N$ of pure strategies. The payoff matrices $\left(a_{i j}\right)_{i \in M, j \in N}$ of player 1 and $\left(b_{i j}\right)_{i \in M, j \in N}$ of player 2 are denoted by $A$ and $B$ respectively. If the players choose mixed strategies $p \in \Delta_{M}$ and $q \in \Delta_{N}$, the payoff for player 1 is $p A q$ and the payoff for player 2 is $p B q$. An equilibrium of this game, which we denote by $(A, B)$, is a strategy pair $(p, q)$ such that

$$
\begin{aligned}
p A q & \geq p^{\prime} A q \text { for all } p^{\prime} \in \Delta_{M} \\
\text { and } \quad p B q & \geq p B q^{\prime} \quad \text { for all } q^{\prime} \in \Delta_{N} .
\end{aligned}
$$

For a strategy $p$ of player 1 , the set $C(p)=\left\{i \in M \mid p_{i}>0\right\}$ is called the carrier of $p$ while

$$
P B_{2}(p):=\left\{j \in N \mid p B e_{j} \geq p B e_{k} \text { for all } k \in N\right\}
$$

is the set of pure best replies of player 2 to $p$. For a strategy $q$ of player 2 , the sets $C(q)$ and $P B_{1}(q)$ are defined in a similar way. It is well-known that $(p, q)$ is an equilibrium of the game $(A, B)$ if and only if $C(p) \subset P B_{1}(q)$ and $C(q) \subset P B_{2}(p)$.

In this paper pairs $(I, J)$, where $I \subset M$ and $J \subset N$ play a prominent role.

Definition A pair $(I, J)$ corresponds to a strategy pair $(p, q)$ if

$$
C(p) \subset I \subset P B_{1}(q) \text { and } C(q) \subset J \subset P B_{2}(p) .
$$

We also say that the pair $(p, q)$ corresponds to the pair $(I, J)$.

\subsection{Perturbed games}

A perturbation for player 1 is a vector $\delta=\left(\delta_{i}\right)_{i \in M}$ with $\delta_{i} \geq 0$ and $\sum_{i \in M} \delta_{i} \leq 1$. The collection of such perturbations is denoted by $\mathscr{D}$. Similarly we can define the collection $\mathscr{E}$ of perturbations $\varepsilon=\left(\varepsilon_{j}\right)_{j \in N}$ for player 2. A pair $(\delta, \varepsilon)$ of perturbations is also called a perturbation.

A perturbation $(\delta, \varepsilon)$ induces a perturbed game $(A, B, \delta, \varepsilon)$. In this game player 1 is only allowed to play a strategy pair in the restricted strategy space $\Delta_{M}(\delta):=$ $\left\{p \in \Delta_{M} \mid p_{i} \geq \delta_{i}\right.$ for all $\left.i \in M\right\}$, while player 2's choices are restricted to the set $\Delta_{N}(\varepsilon)$ that is defined in a similar way. The payoff functions and equilibria of this perturbed game are defined in the obvious way. The collection of equilibria of the perturbed game $(A, B, \delta, \varepsilon)$ is denoted by $E(A, B, \delta, \varepsilon)$. 
In order to characterize the equilibria of the perturbed game $(A, B, \delta, \varepsilon)$ we introduce for player 1 the so-called $\delta$-carrier $C_{\delta}(p):=\left\{i \in M \mid p_{i}>\delta_{i}\right\}$. Analogously one can define the $\varepsilon$-carrier of a strategy $q$ of player 2 . The following characterization, which plays an important role in this paper, can already be found in Lemma 2.3 in Vermeulen (1996).

Lemma 1 The strategy pair $(p, q)$ is an equilibrium of the perturbed game $(A, B, \delta, \varepsilon)$ if and only if the $\delta$-carrier of $p$ is a subset of $P B_{1}(q)$ and the $\varepsilon$-carrier of $q$ is a subset of $\mathrm{PB}_{2}(p)$.

\subsection{Stable sets}

Notice that the choice $\delta=0$ (the null element of the vector space $\mathbb{R}^{M}$ ) and $\varepsilon=0$ returns the original bimatrix game $(A, B)$.

Definition A closed set $S$ in $\Delta_{M} \times \Delta_{N}$ of strategy pairs is called a KM-set if for each neighborhood $V$ of $S$ there exists a number $\eta>0$ such that $V \cap E(A, B, \delta, \varepsilon)$ is not empty whenever $\|(\delta, \varepsilon)\|<\eta$. A minimal KM-set - minimal with respect to set inclusion - is called KM-stable.

Jansen et. al. (1994) showed that

Proposition 1 Every KM-set of a bimatrix game contains a finite KM-set.

\subsection{A result on sensitivity analysis}

An important ingredient of this paper is a result of Cook, Gerards, Schrijver and Tardos on the sensitivity of the solution set of a system of linear inequalities to right-hand perturbations of the system. We will use the following simple version of this result.

Let $\mathscr{b} x \geq \mathscr{B} d$ be a system of linear inequalities and let $\varphi(d):=\{x \mid \mathscr{C x} \geq$ $\mathscr{B} d\}$ be the solution set of this system given $d$.

Proposition 2 There exists a constant $K>0$ such that

$$
d_{H}(\varphi(d), \varphi(e)) \leq K\|d-e\|
$$

for all vectors $d$ and e such that $\varphi(d)$ and $\varphi(e)$ are not empty.

\section{Geometry of the equilibrium correspondence}

Lemma 1 shows that sets of pure strategies are important in the analysis of equilibria. That this is not just idle talk is exemplified in the following.

Let $I \subset M$ be a set of pure strategies of player 1 and let $J \subset N$ be a set of pure strategies of player 2. With these two sets of pure strategies we can associate a subset $S_{I J}$ of the product $\Delta_{M} \times \mathscr{D}$ of the strategy space $\Delta_{M}$ and the 
collection $\mathscr{D}$ of perturbations. This set $S_{I J}$ is formally defined as the collection of solutions $(p, \delta)$ in $\mathbb{R}^{M} \times \mathbb{R}^{M}$ of the system of linear (in)equalities

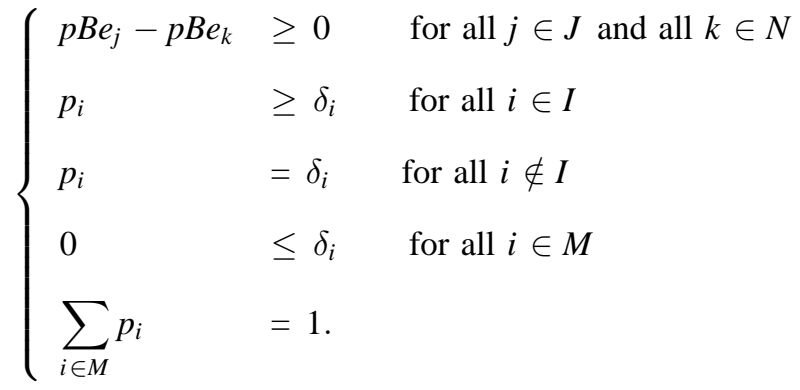

The first group of inequalities states that every pure strategy in $J$ is a best reply against $p$. The second and third group of (in)equalities guarantee that $p$ is an element of $\Delta(\delta)$ and that moreover the $\delta$-carrier of $p$ is a subset of $I$. The fourth and fifth group of (in)equalities are merely added to guarantee that $p$ is indeed a strategy and $\delta$ is indeed a perturbation as soon as $(p, \delta)$ is a solution of the above system of inequalities. (The "missing" inequalities $p_{i} \geq 0$ and $\sum_{i \in M} \delta_{i} \leq 1$ are already implied by the above system.)

Further, note that the variable $p$ only occurs on the left-hand side of the (in)equality signs while the variable $\delta$ only occurs on the right-hand side. Therefore we can define two matrices $\mathscr{C}_{I J}$ and $\mathscr{\mathscr { B }}_{I J}$, whose entries are completely determined by $I$ and $J$ such that the above system of linear inequalities reads as

$$
\mathscr{C}_{I J} p \geq \mathscr{B}_{I J} \delta
$$

The conversion of the smaller-than sign and the equalities in the system $(*)$ into the larger-than signs in the latter system of inequalities can of course easily be established.

In $\Delta_{N} \times \mathscr{E}$ we can analogously define the set $T_{I J}$ by a system of linear inequalities and represent this system by

$$
\mathscr{C}_{I J} q \geq \mathscr{D}_{I J} \varepsilon .
$$

The main advantage of this rather abstract setup is that it enables us to use Proposition 2. Why that is an interesting thing to do becomes clear once we have the following

Lemma 2 Let $(p, q)$ be a strategy pair. Then $(p, q)$ is an equilibrium of the perturbed game $(A, B, \delta, \varepsilon)$ if and only if, for some pair $(I, J),(p, \delta)$ is an element of $S_{I J}$ and $(q, \varepsilon)$ is an element of $T_{I J}$.

Proof. For a pair $(I, J)$

$$
\left\{\begin{array} { l } 
{ ( p , \delta ) \in S _ { I J } } \\
{ ( q , \varepsilon ) \in T _ { I J } }
\end{array} \left\{\begin{array} { l } 
{ \mathscr { C } _ { I J } p \geq \mathscr { B } _ { I J } \delta } \\
{ \mathscr { C } _ { I J } q \geq \mathscr { D } _ { I J } \varepsilon }
\end{array} \Longleftrightarrow \left\{\begin{array}{l}
C_{\delta}(p) \quad \subset I \subset P B_{1}(q) \\
C_{\varepsilon}(q) \quad \subset J \subset P B_{2}(p) .
\end{array}\right.\right.\right.
$$




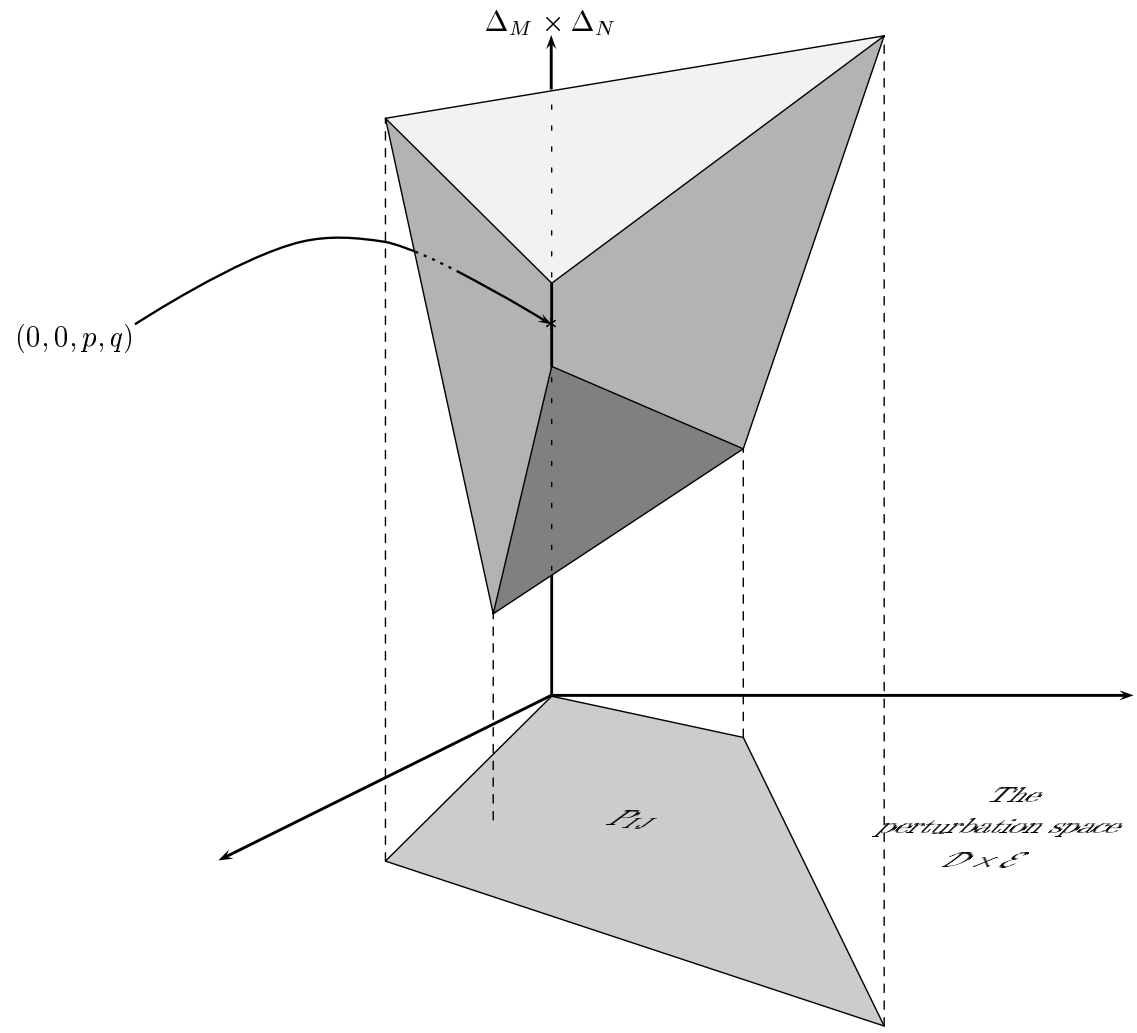

Figure 1

Hence if $(p, \delta) \in S_{I J}$ and $(q, \varepsilon) \in T_{I J}$, then Lemma 1 implies that $(p, q)$ is an equilibrium of the perturbed game $(A, B, \delta, \varepsilon)$. If $(p, q) \in E(A, B, \delta, \varepsilon)$, then take $I=C_{\delta}(p)$ and $J=C_{\varepsilon}(q)$.

The foregoing lemma tells us in fact that we can view the polyhedral set $S_{I J} \times T_{I J}$ as part of the graph of the equilibrium correspondence over $\mathscr{D} \times \mathscr{E}$. In order to formalize this idea we define the correspondence $\varphi_{I J}: \mathbb{R}_{+}^{M} \times \mathbb{R}_{+}^{N} \rightarrow \Delta_{M} \times$ $\Delta_{N}$ as follows: for all $(\delta, \varepsilon) \in \mathbb{R}^{M} \times \mathbb{R}^{N}$,

$$
\varphi_{I J}(\delta, \varepsilon):=\left\{(p, q) \in \Delta_{M} \times \Delta_{N} \mid(p, \delta) \in S_{I J} \text { and }(q, \varepsilon) \in T_{I J}\right\} \subset E(A, B, \delta, \varepsilon) \text {. }
$$

The set of points $(\delta, \varepsilon)$ for which $\varphi_{I J}(\delta, \varepsilon)$ is not empty is denoted by $P_{I J}$. Note that every element $(\delta, \varepsilon)$ of $P_{I J}$ is automatically a perturbation. Furthermore, we can also write $P_{I J}$ as $P_{I J}^{\prime} \times P_{I J}^{\prime \prime}$ with

$$
P_{I J}^{\prime}:=\left\{\delta \mid(p, \delta) \in S_{I J} \text { for some } p\right\} .
$$

Finally, if $(p, q)$ is an equilibrium of the game $(A, B)$ and the pair $(I, J)$ corresponds to $(p, q)$, then $(p, q) \in \varphi_{I J}(0,0)$. So $(0,0) \in P_{I J}$. Also, for all sufficiently small $(\delta, \varepsilon) \in P_{I J}$, the game $(A, B, \delta, \varepsilon)$ has an equilibrium close to $(p, q)$. 
Example. The above figure is to clarify the geometrical intuition underlying the construction just described. The horizontal plane containing the two horizontal axes depicts the perturbation space $\mathscr{D} \times \mathscr{E}$ while the vertical axis stands for the strategy space $\Delta_{M} \times \Delta_{N}$. The block containing the point $(0,0, p, q)$ represents the polyhedral set $S_{I J} \times T_{I J}$. In the figure the pair $(I, J)$ is supposed to correspond to $(p, q)$. By definition of $\varphi_{I J}$ this block is equal to the graph of the correspondence $\varphi_{I J}$.

In Figure 1 the set $S_{I J} \times T_{I J}$ has six extreme points. The domain $P_{I J}$ on which the correspondence $\varphi_{I J}$ is not empty is precisely the convex hull of the projections of the extreme points of $S_{I J} \times T_{I J}$ onto the perturbation space $\mathscr{D} \times \mathscr{E}$. This specific fact will be used in the next section.

\section{Characterization of finite KM-sets}

We exploit the results from the previous section as follows. For a finite set $F$ of equilibria

$$
\mathscr{P}(F):=\left\{P_{I J} \mid(I, J) \text { corresponds to some element of } F\right\} .
$$

Then we have the following

Theorem $1 F$ is a KM-set if and only if the collection $\mathscr{P}(F)$ covers a neighborhood of the zero perturbation $(0,0)$ in $\mathbb{R}_{+}^{M} \times \mathbb{R}_{+}^{N}$.

Proof. (A) Suppose that $\mathscr{P}(F)$ covers a neighborhood of the zero perturbation $(0,0)$. We have to show that $F$ is a KM-set. To this end, take a real number $\zeta>0$. It is sufficient to show that there exists a number $\eta>0$ such that

$$
B_{\zeta}(F) \cap E(A, B, \delta, \varepsilon)
$$

is not empty whenever $\|(\delta, \varepsilon)\|<\eta$.

We will construct an appropriate $\eta$ in two steps. Firstly we take a real number $\eta^{*}>0$ such that every perturbation $(\delta, \varepsilon)$ with

$$
\|(\delta, \varepsilon)\|<\eta^{*}
$$

is an element of some set $P_{I J}$ in $\mathscr{P}(F)$.

Secondly, consider a fixed set $P_{I J}$ in $\mathscr{P}(F)$ and let $(\delta, \varepsilon)$ be a perturbation contained in $P_{I J}$. Since

$$
\begin{aligned}
(p, q) \in \varphi_{I J}(\delta, \varepsilon) & \Longleftrightarrow(p, \delta) \in S_{I J} \text { and }(q, \varepsilon) \in T_{I J} \\
& \Longleftrightarrow\left[\begin{array}{ll}
\mathscr{C}_{I J} & 0 \\
0 & \mathscr{C}_{I J}
\end{array}\right]\left[\begin{array}{l}
p \\
q
\end{array}\right] \geq\left[\begin{array}{ll}
\mathscr{B}_{I J} & 0 \\
0 & \mathscr{V}_{I J}
\end{array}\right]\left[\begin{array}{l}
\delta \\
\varepsilon
\end{array}\right],
\end{aligned}
$$

$P_{I J}$ is precisely the collection of vectors $(\delta, \varepsilon)$ in $\mathbb{R}^{M}$ for which the above system is solvable. So, by Proposition 2 there exists a constant $K_{I J}>0$ such that for any two perturbations $(\delta, \varepsilon)$ and $\left(\delta^{\prime}, \varepsilon^{\prime}\right)$ in $P_{I J}$ 


$$
d_{H}\left(\varphi_{I J}(\delta, \varepsilon), \varphi_{I J}\left(\delta^{\prime}, \varepsilon^{\prime}\right)\right) \leq K_{I J}\left\|(\delta, \varepsilon)-\left(\delta^{\prime}, \varepsilon^{\prime}\right)\right\| .
$$

Now choose a real number $\eta>0$ such that $\eta \leq \eta^{*}$ and moreover

$$
\eta \leq \min \left\{\frac{\zeta}{K_{I J}} \mid P_{I J} \in \mathscr{P}(F)\right\} .
$$

Next, take an arbitrary perturbation $(\delta, \varepsilon)$ with $\|(\delta, \varepsilon)\|<\eta$. We will show that

$$
B_{\zeta}(F) \cap E(A, B, \delta, \varepsilon)
$$

is not empty, which will conclude the first part of the proof.

Since $\eta \leq \eta^{*}$, we know that $(\delta, \varepsilon)$ is an element of some $P_{I J}$ in $\mathscr{P}(F)$. Let $(\tilde{p}, \tilde{q})$ be a point in $F$ that corresponds to the pair $(I, J)$. We will show in particular that

$$
B_{\zeta}(\tilde{p}, \tilde{q}) \cap E(A, B, \delta, \varepsilon)
$$

is not empty. To this end, note that $(\tilde{p}, \tilde{q})$ is an element of $\varphi_{I J}(0,0)$. Furthermore,

$$
d_{H}\left(\varphi_{I J}(\delta, \varepsilon), \varphi_{I J}(0,0)\right) \leq K_{I J}\|(\delta, \varepsilon)\|<K_{I J} \cdot \eta \leq K_{I J} \cdot \frac{\zeta}{K_{I J}}=\zeta .
$$

These two facts combined imply that there is a strategy pair $(p, q)$ in $\varphi_{I J}(\delta, \varepsilon) \subset$ $E(A, B, \delta, \varepsilon)$ whose distance to $(\tilde{p}, \tilde{q})$ is smaller than $\zeta$.

(B) Conversely, suppose that $F$ is a KM-set. We have to show that $\mathscr{P}(F)$ covers a neighborhood of the zero perturbation. So we need to specify a real positive number such that every perturbation whose norm is smaller that this number is an element of some $P_{I J}$ in $\mathscr{P}(F)$. Choosing this particular number requires some effort.

First, take an element $(\tilde{p}, \tilde{q})$ of $F$. By Lemmas 8.5 and 8.6 of Vermeulen (1996) there exist real numbers $\eta>0$ and $\zeta>0$ such that for any $(\delta, \varepsilon)$ with $\|(\delta, \varepsilon)\|<\eta$ and $(p, q) \in \Delta_{M}(\delta) \times \Delta_{N}(\varepsilon)$ with $\|(p, q)-(\tilde{p}, \tilde{q})\|<\zeta$ we have that

$$
\begin{aligned}
& C(\tilde{p}) \subset C_{\delta}(p) \text { and } P B_{1}(q) \subset P B_{1}(\tilde{q}) \\
\text { and } & C(\tilde{q}) \subset C_{\varepsilon}(q) \text { and } P B_{2}(p) \subset P B_{2}(\tilde{p}) .
\end{aligned}
$$

Now, since $F$ is finite, we may suppose that $\eta$ and $\zeta$ do not depend on $(\tilde{p}, \tilde{q}) \in F$.

Furthermore, given the number $\zeta>0$ we can use the assumption that $F$ is a KM-set to obtain a real number $\kappa>0$ such that for any perturbation $(\delta, \varepsilon)$ with $\|(\delta, \varepsilon)\|<\kappa$ we have that

$$
B_{\zeta}(F) \cap E(A, B, \delta, \varepsilon)
$$

is not empty.

Switching back to the main problem, take a perturbation $(\delta, \varepsilon)$ with $\|(\delta, \varepsilon)\|<$ $\min \{\eta, \kappa\}$. We will show that $(\delta, \varepsilon)$ is an element of some set $P_{I J}$ in $\mathscr{P}(F)$. To this end, note that $B_{\zeta}(F) \cap E(A, B, \delta, \varepsilon)$ is not empty since $\|(\delta, \varepsilon)\|<\kappa$. So, 
we can find a point $(\tilde{p}, \tilde{q})$ in $F$ and an equilibrium $(p, q)$ of the perturbed game $(A, B, \delta, \varepsilon)$ such that

$$
\|(\tilde{p}, \tilde{q})-(p, q)\|<\zeta .
$$

Now take $I:=C_{\delta}(p)$ and $J:=C_{\varepsilon}(q)$. We will show (1), that $(\delta, \varepsilon)$ is an element of $P_{I J}$ and (2), that $P_{I J}$ is an element of $\mathscr{P}(F)$.

(1) Since $(p, q)$ is an equilibrium of $(A, B, \delta, \varepsilon)$ we know by Lemma 1 that

$$
C_{\delta}(p) \subset P B_{1}(q) \text { and } C_{\varepsilon}(q) \subset P B_{2}(p) .
$$

So, in particular,

$$
J=C_{\varepsilon}(q) \subset P B_{2}(p)
$$

which, together with the fact that $I=C_{\delta}(p)$ shows that $(p, \delta)$ is indeed an element of $S_{I J}$. Similarly we get that $(q, \varepsilon)$ is an element of $T_{I J}$. So, $(p, q)$ is an element of $\varphi_{I J}(\delta, \varepsilon)$, which immediately implies that $(\delta, \varepsilon)$ is an element of $P_{I J}$.

(2) In order to check that $P_{I J}$ is an element of $\mathscr{P}(F)$ we need to prove that $(I, J)$ corresponds to some element of $F$. Since $\|(\delta, \varepsilon)\|<\eta$ and $\|(\tilde{p}, \tilde{q})-$ $(p, q) \|<\zeta$, we know that

$$
C(\tilde{p}) \subset C_{\delta}(p) \text { and } P B_{1}(q) \subset P B_{1}(\tilde{q}) \text { and } C(\tilde{q}) \subset C_{\varepsilon}(q) \text { and } P B_{2}(p) \subset P B_{2}(\tilde{p}) \text {. }
$$

by the particular choices of $\eta$ and $\zeta$. So, using the fact that $(p, q)$ is an equilibrium of $(A, B, \delta, \varepsilon)$,

$$
C(\tilde{p}) \subset C_{\delta}(p)=I \subset P B_{1}(q) \subset P B_{1}(\tilde{q}) .
$$

A similar line of reasoning yields

$$
C(\tilde{q}) \subset J \subset P B_{1}(\tilde{q})
$$

and we have that $(I, J)$ indeed corresponds to some element of $F$.

\section{The algorithm}

In this section we will discuss how the results from the previous section can be used to check in finite time whether a given finite set $F$ of strategy pairs is a KM-set. We will also elaborate on how this can be used to compute a KM-stable set or even all KM-stable sets.

So, let $F$ be a finite set of strategy pairs of the game $(A, B)$. Now if we want to apply Theorem 1 to check whether $F$ is a KM-set we need to resolve two problems. Firstly, the theorem does not specify a neighborhood. Secondly, given a neighborhood, we would need to check whether $\mathscr{P}(F)$ covers it. The next line of reasoning offers a way out of these problems.

With a set $P_{I J}$ in $\mathscr{P}(F)$ we associate the set $Q_{I J}$ in $\Delta_{M} \times \Delta_{N}$ defined by $Q_{I J}=\left\{(p, q) \in \Delta_{M} \times \Delta_{N} \mid(\lambda p, \mu q) \in P_{I J}\right.$ for some numbers $\lambda>0$ and $\left.\mu>0\right\}$. 
This set is obtained by normalizing the nonzero perturbations in $P_{I J}^{\prime}$ and $P_{I J}^{\prime \prime}$. Now let $\mathscr{Q}(F)$ be the collection of all sets $Q_{I J}$ we can thus construct. Then we get

Theorem $2 \mathscr{Q}(F)$ covers $\Delta_{M} \times \Delta_{N}$ if and only if $\mathscr{P}(F)$ covers a neighborhood of $(0,0)$.

Proof. It is easy to check that $\mathscr{Q}(F)$ covers the strategy space $\Delta_{M} \times \Delta_{N}$ whenever $\mathscr{P}(F)$ covers a neighborhood of $(0,0)$.

To prove the converse statement, suppose that $\mathcal{Q}(F)$ covers $\Delta_{M} \times \Delta_{N}$. Take an element $P_{I J}=P_{I J}^{\prime} \times P_{I J}^{\prime \prime}$ in $\mathscr{P}(F)$. Then we know that $(0,0)$ is an element of $P_{I J}$. So, 0 is an element of $P_{I J}^{\prime}$ and the other 0 is an element of $P_{I J}^{\prime \prime}$. Hence, according to Lemma 4 (which can be found in the Appendix together with the Lemmas 3, 5 and 6), we can associate a number $\eta_{I J}>0$ with $P_{I J}^{\prime}$ and a number $\zeta_{I J}>0$ with $P_{I J}^{\prime \prime}$. Furthermore, since $\mathscr{P}(F)$ is finite, we can take a real number $\eta>0$ smaller than any of the numbers $\eta_{I J}$ and $\zeta_{I J}$ thus found. We will show that the neighborhood $B_{\eta}(0,0)$ is covered by $\mathscr{P}(F)$.

To this end, take a perturbation $(\delta, \varepsilon)$ with $\|(\delta, \varepsilon)\|<\eta$. Assume for the moment that $(\delta, \varepsilon)$ is completely mixed (i.e. $\delta_{i}>0$ for all $i$ and $\varepsilon_{j}>0$ for all $j$ ). Then, since both $\delta$ and $\varepsilon$ are not equal to zero, we can find numbers $\lambda>0$ and $\mu>0$ such that $(\lambda \delta, \mu \varepsilon)$ is an element of $\Delta_{M} \times \Delta_{N}$. So, since $\mathscr{Q}(F)$ is assumed to cover $\Delta_{M} \times \Delta_{N}$ there must be an element $Q_{I J}$ that contains $(\lambda \delta, \mu \varepsilon)$. However, this means that there are numbers $\lambda^{\prime}>0$ and $\mu^{\prime}>0$ such that $\left(\lambda^{\prime} \lambda \delta, \mu^{\prime} \mu \varepsilon\right)$ is an element of $P_{I J}$. Hence, since $\|\delta\|<\eta<\eta_{I J}$ and $\|\varepsilon\|<\eta<\zeta_{I J}$, Lemma 4 states that $(\delta, \varepsilon)$ is an element of $P_{I J}^{\prime} \times P_{I J}^{\prime \prime}=P_{I J}$.

So now we know that $\mathscr{P}(F)$ covers the collection of completely mixed perturbations $(\delta, \varepsilon)$ with $\|(\delta, \varepsilon)\|<\eta$. So, since $\mathscr{P}(F)$ is finite and each element of $\mathscr{P}(F)$ is compact by Lemma $3, \mathscr{P}(F)$ also covers the closure of the collection of completely mixed perturbations $(\delta, \varepsilon)$ with $\|(\delta, \varepsilon)\|<\eta$. This latter set though contains $B_{\eta}(0,0)$.

This theorem reduces our problem (to check in finite time whether a given finite set $F$ of strategy pairs of the game $(A, B)$ is a KM-set) to:

(1) can we compute the collection $\mathscr{Q}(F)$ in finite time and

(2) can we check in finite time whether $\mathcal{Q}(F)$ covers $\Delta_{M} \times \Delta_{N}$.

We will address these questions separately in the next two subsections.

\subsection{Computation of $\mathcal{Q}(F)$}

Given the set $F$ we can calculate for every $(p, q) \in F$ the sets $C(p), P B_{1}(q)$, $C(q)$ and $P B_{2}(p)$ in finite time. So, we can also determine in finite time all pairs $(I, J)$ that correspond to some $(p, q) \in F$. Therefore we can write down all systems of linear inequalities of the form

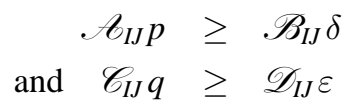


in finite time. This means that we can calculate in finite time a polyhedral description of the set $S_{I J} \times T_{I J}$ for each pair $(I, J)$.

Now a set $P_{I J}$ in $\mathscr{P}(F)$ is simply the orthogonal projection of the corresponding set $S_{I J} \times T_{I J}$ onto the perturbation space $\mathscr{D} \times \mathscr{E}$. (Formally this projection maps onto $\mathbb{R}_{+}^{M} \times \mathbb{R}_{+}^{N}$ but it is easily verified that the set $P_{I J}$ is even a subset of $\mathscr{D} \times \mathscr{E}$.) So, in order to calculate $P_{I J}$ we first need to determine the set

$$
\operatorname{ext}\left(S_{I J} \times T_{I J}\right)=\operatorname{ext}\left(S_{I J}\right) \times \operatorname{ext}\left(T_{I J}\right) .
$$

Given the polyhedral descriptions of $S_{I J}$ and $T_{I J}$ this can also be done in finite time. So, this enables us to calculate the set

$$
\begin{aligned}
G_{I J}:= & \left\{(\delta, \varepsilon) \in \mathbb{R}_{+}^{M} \times \mathbb{R}_{+}^{N} \mid(p, \delta, q, \varepsilon) \in \operatorname{ext}\left(S_{I J} \times T_{I J}\right) \text { for some pair }(p, q)\right. \\
& \left.\in \mathbb{R}^{M} \times \mathbb{R}^{N}\right\} \\
= & \left\{\delta \mid(p, \delta) \in \operatorname{ext}\left(S_{I J}\right) \text { for some } p\right\} \times\left\{\varepsilon \mid(q, \varepsilon) \in \operatorname{ext}\left(T_{I J}\right) \text { for some } q\right\} \\
= & G_{I J}^{\prime} \times G_{I J}^{\prime \prime} .
\end{aligned}
$$

However, it can easily be seen that this set $G_{I J}$ includes $\operatorname{ext}\left(P_{I J}\right)$. So, $P_{I J}$ is the convex hull of $G_{I J}$. Now we have in some sense calculated the set $\mathscr{P}(F)$ in finite time. Each element $P_{I J}$ of $\mathscr{P}(F)$ is stored as the convex hull of the set $G_{I J}$. These sets $G_{I J}$ can indeed be computed in finite time.

Now we turn to the computation of $\mathscr{Q}(F)$. In order to get a representation for each of the elements of $\mathscr{Q}(F)$, take an element $G_{I J}$ that represents a set $P_{I J}$ in $\mathscr{P}(F)$. Define

$$
H_{I J}^{\prime}=\left\{\delta / \sum_{i \in M} \delta_{i} \mid \delta \in G_{I J}^{\prime} \text { and } \delta \neq 0\right\} .
$$

Similarly we define $H_{I J}^{\prime \prime}$. The set $H_{I J}:=H_{I J}^{\prime} \times H_{I J}^{\prime \prime}$ is obviously a subset of the strategy space $\Delta_{M} \times \Delta_{N}$. Moreover, by Lemma $6, Q_{I J}$ is precisely the convex hull of $H_{I J}$. Hence, it is verified that $H_{I J}$ contains the set of extreme points of $Q_{I J}$.

\subsection{The covering problem}

Now we have a representation of each set $Q_{I J}$ in $\mathscr{Q}(F)$ in the form of the set $H_{I J}$. The set $Q_{I J}$ itself is simply the convex hull of $H_{I J}$. So now we can address the second problem. How do we check whether $\mathscr{Q}(F)$ covers $\Delta_{M} \times \Delta_{N}$ ? To do this we need to calculate a system

$$
\mathscr{H}_{I J}\left[\begin{array}{l}
p \\
q
\end{array}\right] \geq b_{I J}
$$

of linear inequalities whose solution set is exactly $Q_{I J}$. Given the set $H_{I J}$ that can also be done in finite time. Given this polyhedral description of the polytopes 
in $\mathcal{Q}(F)$ it is possible to show that the covering problem can be solved in finite time. This is easier to explain in a somewhat more general setting.

Suppose that a polytope $P$ in $\mathbb{R}^{n}$ is given by the system

$$
\mathscr{P} x \geq b
$$

of linear inequalities where $\mathscr{P}$ is an $m \times n$ matrix. Furthermore, suppose that for each $j \in\{1, \ldots, J\}$ a polytope $Q_{j}$ in $\mathbb{R}^{n}$ is given by the system

$$
Q_{j} x \geq c_{j}
$$

of linear inequalities where $\mathcal{Q}$ is a $k_{j} \times n$ matrix. The central question is whether the polytopes $Q_{1}, \ldots, Q_{J}$ cover the polytope $P$. Note first that we may assume w.l.o.g. that $P$ is of full dimension. Otherwise we can restrict the problem to the affine hull of $P$ (in finite time!).

In order to explain how we can check whether $Q_{1}, \ldots, Q_{J}$ cover $P$ we introduce the notion of transversal system.

Definition Given the systems of polyhedral inequalities that describe the polytopes $Q_{1}, \ldots, Q_{J}$ and $P$, a tranversal system over the sets $Q_{1}, \ldots, Q_{J}$ w.r.t. $P$ is a system of linear inequalities of the form

$$
\left\{\begin{array}{cc}
e_{k_{1}} \mathcal{Q}_{1} x & <\left(c_{1}\right)_{k_{1}} \\
& \vdots \\
e_{k_{J}} \mathcal{Q}_{J} x & <\left(c_{J}\right)_{k_{J}} \\
\mathscr{P} x & \geq b .
\end{array}\right.
$$

So, from each system $Q_{j} x \geq c_{j}$ we pick precisely one row, say $k_{j}$, and change the greater-than-or-equal sign into a smaller-than sign. Furthermore we add the inequalities that describe the polytope $P$.

Now it is obvious that $Q_{1}, \ldots, Q_{J}$ cover $P$ if and only if each transversal system is not solvable. This is so if and only if the solution to the linear problem to maximize $y$ subject to

$$
\begin{cases}e_{k_{1}} \mathcal{Q}_{1} x+y & \leq\left(c_{1}\right)_{k_{1}} \\ & \vdots \\ e_{k_{J}} \mathcal{Q}_{J} x+y & \leq\left(c_{J}\right)_{k_{J}} \\ \mathscr{P} x & \geq b\end{cases}
$$

is smaller or equal to zero. This latter statement can be verified in finite time using a simple linear program solver. 
Hence, $\mathcal{Q}(F)$ covers $\Delta_{M} \times \Delta_{N}$ if and only if each transversal system over the set of all proper pairs $(I, J)$ of the systems

$$
\mathscr{H}_{I J}\left[\begin{array}{l}
p \\
q
\end{array}\right] \geq b_{I J}
$$

w.r.t. $\Delta_{M} \times \Delta_{N}$ is empty. According to the above argumentation this can be checked in finite time.

\section{Computation of KM-stable sets and strictly perfect equilibria}

Let $(A, B)$ be a bimatrix game and let $F$ be a finite set of strategy pairs. From Theorem 1 we know how to check whether $F$ is KM-stable or not. $F$ is KM-stable if and only if

(1) $\mathscr{P}(F)$ covers a neighborhood of $(0,0)$, and

(2) there exists no proper subset $G$ of $F$ such that $\mathscr{P}(G)$ covers a neighborhood of $(0,0)$.

Given the set $F$ we know how to check both items in finite time. This gives us an opportunity to compute KM-stable sets. One way to do that works as follows.

Suppose we know that $F$ is a KM-set. Then it is easy to construct a KMstable subset of $F$. Check for each proper subset $G$ of the set $F$ whether it is a $\mathrm{KM}$-set. If none of these sets is a KM-set, then $F$ is KM-stable. If one of these sets is a KM-set, repeat the above procedure with respect to this new set. Since $F$ is finite we will find a KM-stable subset of $F$ in a finite number of steps.

So the only question is whether we can find a finite set $F$ of which we know that it is a KM-set. There is at least one way to find such a set.

Jansen et al. (1994) showed that the set of extreme equilibrium points is a $\mathrm{KM}$-set of the game $(A, B)$. This set can be computed in finite time.

\subsection{Computation of all KM-stable sets}

However, from the formulation of (1) and (2) we can also derive a more general procedure for the computation of KM-sets.

Let $\mathscr{C}$ be a collection of pairs $(I, J)$ with $I \subset M$ and $J \subset N$. With each pair $(I, J)$ we can associate a set $P_{I J}$. Let $\mathscr{P}$ be the collection of sets of the form $P_{I J}$ with $(I, J) \in \mathscr{C}$. Then we can check in finite time whether

(1) $\mathscr{P}$ covers a neighborhood of $(0,0)$, and

(2) $\mathscr{P}$ has no proper subcollection that covers a neighborhood of $(0,0)$.

Now suppose that we do have such a set $\mathscr{C}$ (i.e. its associated set $\mathscr{P}$ covers $(0,0)$ and no proper subcollection of $\mathscr{P}$ does so). Then we can construct a set $F$ as follows. 
(1) Take a pair $(I, J)$ in $\mathscr{C}$. Since no proper subset of $\mathscr{P}$ covers a neighborhood of $(0,0)$ we know that $(0,0)$ is an element of $P_{I J}$. Therefore it is possible to compute an equilibrium $\left(p_{I J}, q_{I J}\right)$ in $\varphi_{I J}(0,0)$.

(2) Once this is done for every set $P_{I J}$ in $\mathscr{P}$ we know that the finite set

$$
F:=\left\{\left(p_{I J}, q_{I J}\right) \mid(I, J) \in \mathscr{C}\right\}
$$

is a $\mathrm{KM}$-set. Hence, we can find a KM-stable subset of $F$ using the procedure described above.

The assertion now is that every KM-stable set can be found in this way given that we pick the right equilibria in step (1) and throw out the right equilibria in the selection procedure. In this sense it is possible to compute all KM-stable sets of a given bimatrix game. Notice that generically speaking each set $P_{I J}$ admits only one choice. So, generically it is possible to actually write down all KM-stable sets.

\subsection{Computation of strictly perfect equilibria}

In this section we will discuss how the results from the previous section can be used to check in finite time whether or not a given strategy pair is a strictly perfect equilibrium. It will also enable us to compute one (if existing) or even all strictly perfect equilibria.

Definition A strategy pair $(p, q)$ in $\Delta_{M} \times \Delta_{N}$ is called a strictly perfect equilibrium if for each neighborhood $V$ of $(p, q)$ there exists a number $\eta>0$ such that

$$
V \cap E(A, B, \delta, \varepsilon)
$$

is not empty whenever $\|(\delta, \varepsilon)\|<\eta$.

Obviously a strategy pair $(p, q)$ is a strictly perfect equilibrium if and only if $\{(p, q)\}$ is a KM-set. So, if we want to find a strictly perfect equilibrium, we can use the following procedure. First calculate all sets $\mathscr{P}$ that are minimally covering. Secondly, check whether the sets $\varphi_{I J}(0,0)$ associated with $\mathscr{P}$ have a non-empty intersection. If yes, each element of this intersection is a strictly perfect equilibrium. If no, then this set $\mathscr{P}$ only generates KM-stable sets (at least one) that consists of more than one element.

A more efficient algorithm can be based on a result of Jansen et al. (1994). In fact they prove that a bimatrix game possesses a strictly perfect equilibrium if and only if there exists an extreme equilibrium that is strictly perfect. Now there are algorithms that calculate all extreme equilibria of a bimatrix game. Given these extreme equilibria, it can be checked whether any of them is strictly perfect using the algorithm of the previous section. The result of Jansen et al. now guarantees that either we find a strictly perfect equilibrium, or we find out that the game in question does not have a strictly perfect equilibrium. 


\section{Appendix A}

In this section we will go into the details of the analysis of the systems of linear inequalities that define the various sets used in the paper. First we will show

Lemma 3 The sets $S_{I J}, T_{I J}, P_{I J}^{\prime}, P_{I J}^{\prime \prime}$ and $P_{I J}$ are polytopes.

Proof. First consider the set $S_{I J}$. By definition this set is the collection of points $(p, \delta)$ that satisfy the system $\mathscr{C}_{I J} p \geq \mathscr{B}_{I J} \delta$ of linear inequalities. So $S_{I J}$ is polyhedral. Furthermore, the points in $S_{I J}$ particularly satisfy

$$
\left\{\begin{array}{lll}
p_{i} & \geq \delta_{i} \quad \text { for all } i \in I \\
p_{i} & =\delta_{i} \quad \text { for all } i \notin I . \\
0 & \leq \delta_{i} \quad \text { for all } i \in M \\
1 & \geq \sum_{i \in M} \delta_{i} \\
\sum_{i \in M} p_{i} & =1 .
\end{array}\right.
$$

This clearly shows that $S_{I J}$ is bounded. Hence, $S_{I J}$ (and $T_{I J}$ for similar reasons) is a polytope.

Furthermore, $P_{I J}^{\prime}$ is the set of $\delta$ such that $(p, \delta) \in S_{I J}$ for some $p$. Hence $P_{I J}^{\prime}$ is the image of the polytope $S_{I J}$ under the linear map $(p, \delta) \mapsto \delta$. Hence, $P_{I J}^{\prime}$ (and $P_{I J}^{\prime \prime}$ also) is a polytope. Finally, $P_{I J}$ must be a polytope since it is the product of the polytopes $P_{I J}^{\prime}$ and $P_{I J}^{\prime \prime}$.

Now we can prove the following lemma. Obviously we can do something similar for $P_{I J}^{\prime \prime}$. Its associated positive real number is denoted by $\zeta_{I J}$.

Lemma 4 Suppose that 0 is an element of $P_{I J}^{\prime}$. Then there exists a real number $\eta_{I J}>0$ such that any point $\delta$ with $\|\delta\|<\eta_{I J}$ is an element of $P_{I J}^{\prime}$ whenever $\lambda \delta$ is an element of $P_{I J}^{\prime}$ for some real number $\lambda>0$.

Proof. Since $P_{I J}^{\prime}$ is a polytope by Lemma 3, we can find a system

$$
\mathscr{F} \delta \geq b
$$

of linear inequalities whose solution set equals $P_{I J}^{\prime}$. Now since 0 is an element of $P_{I J}^{\prime}$ by assumption, we know that $0=\mathscr{F} 0 \geq b$. Let $K$ be the collection of numbers $k$ for which $0>b_{k}$, where $b_{k}$ is the $k$-th coordinate of $b$. Then for each $k \in K$ we can take a number $\eta_{k}>0$ such that $e_{k} \mathscr{F} \delta>b_{k}$ whenever $\|\delta\|<\eta_{k}$. Choose $\eta_{I J}>0$ smaller than any number $\eta_{k}$. Let $\delta$ be a point with $\|\delta\|<\eta_{I J}$ and suppose that $\lambda>0$ is such that $\lambda \delta$ is element of $P_{I J}^{\prime}$. Then, since $\|\delta\|<\eta_{I J}$ we know that

$$
e_{k} \mathscr{F} \delta>b_{k}
$$

for every $k$ in $K$. So we only need to show that $e_{h} \mathscr{F} \delta \geq b_{h}$ for $h \notin K$. Since $h$ is not an element of $K$, we know that $0=b_{h}$. Further, since $\lambda \delta$ is an element 
of $P_{I J}^{\prime}$ we also know that $\mathscr{F}(\lambda \delta) \geq b$. So, in particular, $e_{h} \mathscr{F}(\lambda \delta) \geq b_{h}=0$ and (since $\lambda>0$ ) we can calculate that

$$
e_{h} \mathscr{F} \delta=\lambda^{-1} e_{h} \mathscr{F}(\lambda \delta) \geq \lambda^{-1} \cdot 0=0=b_{h} .
$$

This shows that $\mathscr{F} \delta \geq b$ and $\delta$ is an element of $P_{I J}^{\prime}$.

$\triangleleft$

Lemma 5 Let $r$ and $s$ be two points in $\mathbb{R}^{M}$ and suppose that $p=\rho r+(1-\rho) s$ for some $0 \leq \rho \leq 1$. Suppose further that $a$ and $b$ are two positive real numbers. Then there is a positive multiple of $p$ that is also a convex combination of ar and bs.

Proof. We have to show that there are numbers $0 \leq \kappa \leq 1$ and $\zeta>0$ such that

$$
\zeta p=\kappa(a r)+(1-\kappa)(b s) .
$$

Take

$$
\kappa:=\frac{b \rho}{b \rho+a(1-\rho)} \quad \text { and } \zeta:=\frac{a b}{b \rho+a(1-\rho)}
$$

It easily follows that $0 \leq \kappa \leq 1$ and $\zeta>0$ since $a$ and $b$ are positive and $0 \leq \rho \leq 1$. Moreover,

$$
\begin{aligned}
\kappa(a r)+(1-\kappa)(b s) & =\frac{b \rho}{b \rho+a(1-\rho)}(a r)+\frac{a(1-\rho)}{b \rho+a(1-\rho)}(b s) \\
& =\frac{a b}{b \rho+a(1-\rho)}(\rho r+(1-\rho) s)=\zeta(\rho r+(1-\rho) s)=\zeta p . \triangleleft
\end{aligned}
$$

Lemma 6 The set $Q_{I J}$ is the convex hull of the set $H_{I J}$.

Proof. We will exploit the decomposition of $P_{I J}$ into the product $P_{I J}^{\prime} \times P_{I J}^{\prime \prime}$. Notice that this enables us to write

$$
Q_{I J}=Q_{I J}^{\prime} \times Q_{I J}^{\prime \prime}
$$

where $\quad Q_{I J}^{\prime}:=\left\{p \in \Delta_{M} \mid \lambda p \in P_{I J}^{\prime}\right.$ for some number $\left.\lambda>0\right\}$

and $Q_{I J}^{\prime \prime}$ is defined analogously. It suffices to show that $Q_{I J}^{\prime}$ is the convex hull of $H_{I J}^{\prime}$. To this end, notice that $P_{I J}^{\prime}$ is the collection of points $\delta$ in $\mathbb{R}^{M}$ for which the system

$$
\mathscr{C p} \geq \mathscr{B} \delta
$$

has a solution $p$. So, any point $\delta$ in $P_{I J}^{\prime}$ at least has to satisfy the inequalities

$$
\delta_{i} \geq 0 \quad \text { for all } i \in M \text {. }
$$

So, since $G_{I J}^{\prime}:=\left\{\delta \mid(p, \delta) \in \operatorname{ext}\left(S_{I J}\right)\right.$ for some $\left.p\right\}$ is a subset of $P_{I J}^{\prime}$ we know that $G_{I J}^{\prime}$ is a subset of the non-negative orthant of $\mathbb{R}^{M}$. From this it easily follows that $H_{I J}^{\prime}$ is a subset of $Q_{I J}^{\prime}$ since $\sum_{i \in M} \delta_{i}$ is automatically positive for each non-zero element $\delta$ in the non-negative orthant. 
A. First we will show that $Q_{I J}^{\prime}$ is convex. To this end, take two points $r$ and $s$ in $Q_{I J}^{\prime}$. We will show that a convex combination $\rho r+(1-\rho) s$ is also an element of $Q_{I J}^{\prime}$.

Since $r$ and $s$ are points in $Q_{I J}^{\prime}$ we can take $\lambda>0$ and $\mu>0$ such that $\lambda r$ and $\mu s$ are elements of $P_{I J}^{\prime}$. Furthermore, from Lemma 5 it follows that there exists a positive multiple of $\rho r+(1-\rho) s$ that is also a convex combination of $\lambda r$ and $\mu s$. However, a convex combination of $\lambda r$ and $\mu s$ must be an element of $P_{I J}^{\prime}$ since $P_{I J}^{\prime}$ is convex. Hence, $\rho r+(1-\rho) s$ is a positive multiple of an element in $P_{I J}^{\prime}$ and therefore an element of $Q_{I J}^{\prime}$.

B. Conversely, let $p$ be an element of $Q_{I J}^{\prime}$. We have to show that $p$ is a convex combination of elements in $H_{I J}^{\prime}$. To this end, notice that $\lambda p$ is an element of $P_{I J}^{\prime}$ and therefore a convex combination of the elements of $G_{I J}^{\prime}$. By changing the multiple $\lambda$ if necessary we can assume that it is even a convex combination of the non-zero elements of $G_{I J}^{\prime}$.

Suppose that $\lambda p=\rho r+(1-\rho) s$, with $r$ and $s$ both in the non-negative orthant and not equal to 0 . Write

$$
a:=\left(\sum_{i \in M} r_{i}\right)^{-1} \text { and } b:=\left(\sum_{i \in M} s_{i}\right)^{-1} .
$$

Then we can take $\kappa$ and $\zeta$ as in Lemma 5 and we get that

$$
\zeta \lambda p=\kappa(a r)+(1-\kappa)(b s) .
$$

It is sufficient to show that $\zeta \lambda=1$. To this end notice that

$$
\begin{aligned}
\zeta \lambda & =\zeta \lambda \sum_{i \in M} p_{i}=\sum_{i \in M} \zeta \lambda p_{i}=\sum_{i \in M}[\kappa(a r)+(1-\kappa)(b s)]_{i} \\
& =\kappa a \sum_{i \in M} r_{i}+(1-\kappa) b \sum_{i \in M} s_{i}=1 .
\end{aligned}
$$

Now the statement follows by induction to the number of non-zero elements of $G_{I J}^{\prime}$ that are used to write $\lambda p$ as a convex combination.

\section{References}

Aggarwal, V.: On the generation of all equilibrium points for bimatrix games through the LemkeHowson algorithm. Mathematical Programming 1, 232-234 (1973)

Blume, L.E., Zame, W.R.: The algebraic geometry of perfect and sequential equilibrium. Econometrica 62, 783-794 (1994)

Cook, W., Gerards, A.M.H., Schrijver, A., Tardos, E.: Sensitivity theorems in integer linear programming. Mathematical Programming 34, 48-61 (1986)

Elzen, A.H., van den, Talman, A.J.J.: A procedure for finding Nash equilibria in bi-matrix games. Zeitschrift für Operations Research - Methods and Models of Operations Research 35, 27-43 (1991)

Hillas, J.: On the definition of the strategic stability of equilibria. Econometrica 58, 1365-1391 (1990) 
Hillas, J., Vermeulen, D., Jansen, M.: On the finiteness of stable sets. International Journal of Game Theory 26, 275-278 (1997)

Jansen, M.J.M., Jurg, A.P., Borm, P.E.M.: On strictly perfect sets. Games and Economic Behaviour 6, 400-415 (1994)

Harsanyi, J.C.: The tracing procedure: a Bayesian approach to defining a solution for $n$-person noncooperative games. International Journal of Game Theory 4, 61-94 (1975)

Herings, J.-J., Peeters, R.: A differentiable homotopy to compute Nash equilibria of $n$-person games. Research Memorandum 99/33, Faculty of Economics and Business Administration, University of Maastricht, The Netherlands (1999)

Kohlberg, E., Mertens, J.F.: On strategic stability of equilibria. Econometrica 54, 1003-1037 (1986)

Krohn, I., Moltzahn, S., Rosenmüller, P. Sudhölter, J., Wallmeier, H.M.: Implementing the modified LH algorithm. Applied Mathematics and Computation 45, 31-72 (1991)

Lemke, E., Howson, T.: Equilibrium points in bimatrix games. SIAM Journal of Applied Mathematics 12, 413-423 (1964)

Mertens, J.F.: Stable equilibria-a reformulation. Part I: Definitions and basic properties. Mathematics of Operations Research 14, 575-625 (1989)

Mertens, J.F.: Stable equilibria-a reformulation. Part II: Discussion of the definition and further results. Mathematics of Operations Research 16, 694-753 (1991)

Myerson, R.B.: Refinements of the Nash equilibrium concept. International Journal of Game Theory 7, 73-80 (1978)

Nash, J.F.: Equilibrium points in $n$-person games. Proceedings from the National Academy of Science, USA 36, 48-49 (1950)

Okada, A.: On stability of perfect equilibrium points. International Journal of Game Theory 10, 67-73 (1981)

Rosenmüller, J.: On a generalization of the Lemke-Howson algorithm to noncooperative $n$-person games. SIAM Journal of Applied Mathematics 21, 73-79 (1971)

Selten, R.: Reexamination of the perfectness concept for equilibrium points in extensive games. International Journal of Game Theory 4, 25-55 (1975)

Talman, A.J.J., Yang, Z.: A simplicial algorithm for computing proper Nash equilibria of finite games. CentER DP 9418, Tilburg University, The Netherlands (1994)

Vermeulen, A.J.: Stability in non-cooperative game theory. PhD Thesis, Department of Mathematics, University of Nijmegen, The Netherlands (1996)

Wilson, R.: Computing simply stable equilibria. Econometrica 60, 1039-1070 (1992)

Winkels, H.M.: An algorithm to determine all equilibrium points of a bimatrix game. In: Moeschlin, O., Pallaschke, D., (eds.) Game theory and related topics, pp. 137-148. Amsterdam: North Holland 1979

Yamamoto, Y.: A pathfollowing procedure to find a proper equilibrium of finite games. International Journal of Game Theory 22, 249-259 (1993) 